\title{
Modelling Ultraviolet Threats
}

\author{
James I.* \\ Cranfield University, Shrivenham, Wiltshire, UK
}

\begin{abstract}
Electro-optically (EO) guided surface to air missiles (SAM) have developed to use Ultraviolet (UV) wavebands supplementary to the more common Infrared (IR) wavebands. Missiles such as the US Stinger have been around for some time but are not considered a proliferation risk. The Chinese FN-16 and Russian SA-29 (Verba) are considered a much higher proliferation risk. As a result, models of the missile seekers must be developed to understand the characteristics of the seeker and the potential performance enhancement that are included. Therefore, the purpose of this paper is to introduce the steps that have been taken to characterise and model these missiles. It begins by outlining some of the characteristics of the threats, the key elements of a UV scene, the potential choice of waveband for a detector, the initial modelling work to represent the UV detector of the missile and presents initial results. The modelling shows that the UV detection range of a typical aircraft is dependent on both the size of the aircraft and its reflectivity. However, the strength of this correlation is less than expected. As a result, further work is required to model more seeker types and to investigate what is causing the weak correlations found in these initial investigations. In addition, there needs to be further study of the sensitivities of the model to other variables, such as the modelled detectivity of the detector and the signal to noise ratio assumed. Overall, the outcome of this work will be to provide specifications for aircraft size and reflectivity that limit the effectiveness of the UV channels.
\end{abstract}

Keywords: Ultraviolet, UV, missile, model, aircraft

\section{INTRODUCTION}

Since the introduction of EO SAM in the 1960s, there has been a continuous race between the missile manufacturers, who seek to create a missile that can engage and hit a target aircraft, and the aircraft protection engineers, who endeavor to protect the aircraft through whatever means possible. Once they have been able to against a particular missile, the onus is on the manufacturers to improve it or produce a different variant to ensure that it, once again, is able to engage and hit the target. This is a classic arms race and in the EO SAM environment, this has involved IR seekers and IR Countermeasures (CM) such as flares, lamps and lasers. Many iterations of this arms race have occurred, leading to more complex CM, as well as the introduction of Counter-Countermeasures (CCM) within the missile to reject these flare CM. One method for mitigating many of the CM currently deployed is to change the waveband in which the missile gathers information about the scene. This has been recently done with the introduction of EO SAM that operate with a UV detector.

This creates a number of new problems for the platform protection engineer that must be solved, as shown at Table 1, which the information that the engineer requires and some example methods by which information can be gathered in order to overcome these problems. It should be noted that this list is not exhaustive and that it does not attempt to be realistic about what may be possible. However, it is useful in providing insight into what (say why it is good despite its limitations). In the absence of precise information, the platform protection engineer can opt to build a model of the system, which must be at a suitable level of complexity, in order to provide information about any apparent dependence on specific variables or sensitivities to other factors.

\footnotetext{
*i.james@cranfield.ac.uk
} 
Table 1 New challenges for the platform protection engineer

\begin{tabular}{|l|l|l|}
\hline \multicolumn{1}{|c|}{ Problem } & \multicolumn{1}{|c|}{ Information Required } & \multicolumn{1}{|c|}{ Method } \\
\hline $\begin{array}{l}\text { Understand the capability } \\
\text { of the detector }\end{array}$ & Characteristics of the detector & $\begin{array}{l}\text { Literature search, investigation } \\
\text { of sample device(s) }\end{array}$ \\
\hline $\begin{array}{l}\text { Understand the nature of } \\
\text { the scene }\end{array}$ & $\begin{array}{l}\text { Background radiance levels at } \\
\text { various times of day and year at } \\
\text { various locations and various } \\
\text { metrological conditions. }\end{array}$ & $\begin{array}{l}\text { Literature search, long term } \\
\text { sampling, physics based } \\
\text { models }\end{array}$ \\
\hline $\begin{array}{l}\text { Understand the aircraft } \\
\text { signature }\end{array}$ & $\begin{array}{l}\text { Reflectivity of surface finish, links to } \\
\text { background signature, scattering } \\
\text { effects, typical engagement ranges }\end{array}$ & $\begin{array}{l}\text { Investigate potential paint and } \\
\text { surface finishes and test them }\end{array}$ \\
\hline $\begin{array}{l}\text { Understand the new } \\
\text { capabilities of the missile }\end{array}$ & $\begin{array}{l}\text { How the missile seeker tracks, how it } \\
\text { responds to CM, how the UV } \\
\text { waveband is used (tracking or guard) }\end{array}$ & $\begin{array}{l}\text { Produce and test models of the } \\
\text { various missile seeker options, } \\
\text { conduct sensitivity testing }\end{array}$ \\
\hline
\end{tabular}

It is the creation of suitable models of EO SAM missile seekers, operating in the UV waveband, which is the subject of this paper. The purpose of the research being undertaken is to provide insight into the detection ranges that can be expected of a typical missile UV detector that will inform the signature control measures required to mitigate this. Also included are some of the initial results. This paper will begin with examination of the potential threat missiles followed by an introduction to the UV scene and its peculiar characteristics. A proof of concept study into whether these missiles and the scene can be modelled will be completed through examination of the method by which models have been created and tested, discussion of the results and conclusions will follow.

\section{THE POTENTIAL THREATS}

The potential threats that are detailed in the introduction include the Chinese FN-16 and the Russian SA-29 (Verba) missiles and these warrant some explanation. The information about these missiles has been is obtained from various open sources including the Internet and airshow brochures. Additional information for the SA-29 has been derived from interpretation of two patents that are assumed to be linked to the missile [1] [2]. Both threats are believed to contain a UV band detector (or detectors) and this is used, primarily, to assist with the discrimination of the aircraft from its background and, more importantly, from CM. It is expected that this is possible because the aircraft blocks a significant portion of the UV background during daylight hours. However, some other, novel technology is expected to be present in these missile threats and this is likely affect the models created. Both the FN-16 and the SA-29 use scan patterns that require further consideration. Neither is a standard reticle based conscan or spinscan seeker and therefore care must be taken to understand the advantage that this gives the missile seeker. Some of the key technologies, particularly the detector arrangement and scan patterns contained within these missile seekers, are discussed below.

\subsection{Multi slit detector}

The SA-29 (Verba) has a peculiar detector arrangement, which consists of detectors operating in three distinct wavebands [2]. These are Short Wave IR (SWIR), Wide Band IR (WBIR) and UV. The precise definitions of these are not provided by the available literature. However, it is assumed that SWIR refers to the portion of the IR spectrum from 1.4-3 $\mu \mathrm{m}$. Similarly, the WBIR is ill defined but, given that it is distinct from the SWIR and Medium Wave IR, it is assumed some combination of both $(1.4-8 \mu \mathrm{m})$. It is judged that the precise waveband is inconsequential for the current study because the ability for the missile to track with the IR detector is all that is important in the initial phases. The UV is assumed to be UVA and UVB as described in Section 3. In addition to this, it is noted that the arrangement of the detector elements is most peculiar in that for each detector type there appears to be three slit detector elements arranged radially from a central point, as shown at Figure 1. It is judged that the specific arrangement of detector elements should enable this seeker to derive precise information about the motion of the target aircraft across its Field of View (FOV) and should enable a broad suite of CCM to be implemented. This design appears most suited to a spinscan arrangement with a small center null. This novel arrangement is included in the model of the SA-29 (Verba) including some estimation of likely CCM, which makes use of the detector arrangement. 


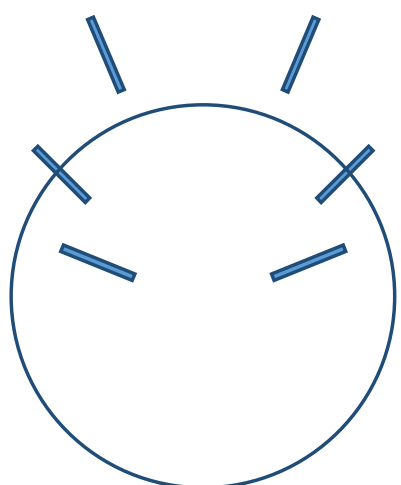

Figure 1 SA-29 (Verba) slit detector arrangement (in blue) with circular conscan locus of aircraft shown

\subsection{Rosette scan}

The FN-16 is marketed as containing a rosette-scanning seeker. This type of scan is produced when a folded, cassegrain telescope has two contra-rotating elements that are canted at an angle from each other. These elements must rotate at different speeds. This arrangement can prescribe multiple scan patterns, such as spirals or lines, but a particular set of characteristics gives rise to a repeating rosette scan pattern. Much work has been done on producing optimal rosette scan patterns for missile seekers [3][4]. Given this, and the available information, it is assumed that the rosette scan pattern used within the FN-16 is that which is given in the literature [5], shown at Figure 2, although this may not be associated with the FN-16 missile. The precise parameters of the rosette scan should not affect the detectivity of the missile seeker.

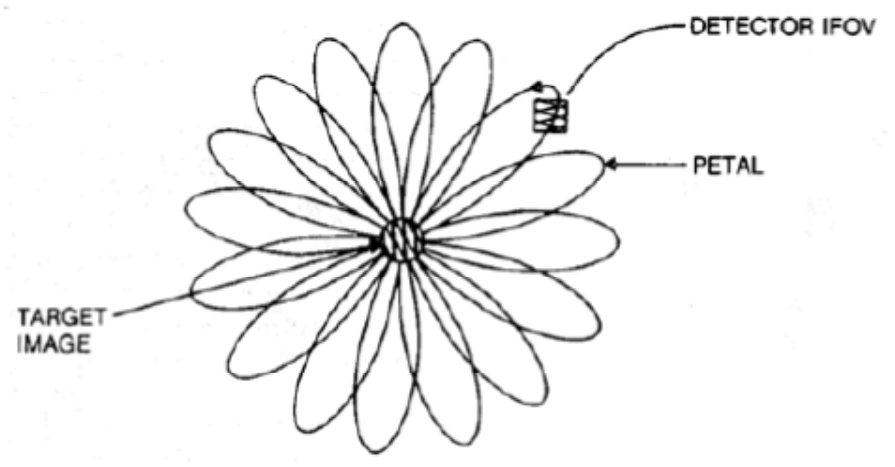

Figure 2 Rosette scan pattern [5]

The rosette scan allows a missile designer to use a small Instantaneous FOV (IFOV) that is then scanned over a much larger FOV at a high speed. Samples of this FOV are taken at high frequency and the location of each sample, described as an $\mathrm{x}$ and $\mathrm{y}$ or $\mathrm{r}$ and $\theta$ co-ordinate in the FOV is then known. This information can be used to provide a steering command to the missile seeker or body to track any object within the FOV. The small IFOV inherent within the rosette scan ensures that rapidly separating, dispensable objects such as flare CM, which will leave the IFOV quickly, do not easily capture the seeker. Furthermore, the scan pattern ensures that the object in the center of the FOV is scanned most regularly and the extremes of the FOV are scanned less frequently. This gives the missile seeker greater opportunity to interrogate, and maintain track upon, the target and will limit its susceptibility to dispensable CM.

\subsection{Other key technology}

It is purported that both the SA-29 (Verba) and the FN-16 use pseudo-imaging capabilities [6]. Therefore, it can be envisaged that the data from a set time period from either detector arrangement could be stored as a 'frame' and that standard image processing algorithms can be applied to a series of such 'frames' to provide information on any changes from one frame to another. This particular capability is difficult to estimate and is beyond the scope of this paper. However, assumptions need to be made about the detector material used to detect the UV energy. A typical detector material is Gallium Phosphate, although Silicon can also be used. However, the actual material is less important to define than the performance of that material in terms of its detectivity and the signal to noise ratio inherent therein as these will define the range at which the detector can discriminate objects. The UV wavelengths that can be detected are defined by 
the detector material used and can be limited further by filtering. An assumption can be made about the precise wavelengths that are likely to be used. This is discussed further in the next section.

\section{THE UV SCENE}

\subsection{Background and target}

The background level of UV radiation is one that humans, are familiar with, causing burns if outside in the sun without protection. Similarly, UV can cause degradation of materials, such as plastic, when left outside for long periods. Humans are protected from UV by sunscreen and shade. It is difficult to define this UV environment, as it cannot be seen. It is composed of radiation from the sun and, therefore, there is little energy present outside of daylight hours. It is also strongly absorbed by the atmosphere. However, owing to the scattering of the UV radiation from the sun, which is not strongly absorbed, and the long path length through the atmosphere (the scattering medium), the sky appears at a relatively uniform radiation. This means that when the sky is observed in the UV there is often no variation of intensity whether the sky has clouds present or not. Aircraft, and other objects, can easily be discriminated against this ideal background.

The wavelengths associated with UV are well defined, ranging from $10 \mathrm{~nm}$ through to $400 \mathrm{~nm}$. However, the regions that are useful for detection of aircraft are much tighter. The definitions of the bands are shown at Table 2.

Table 2 UV wavebands

\begin{tabular}{|c|c|}
\hline UV Waveband & Wavelength (nm) \\
\hline UVA & $315-400$ \\
\hline UVB & $280-315$ \\
\hline UVC & $100-280$ \\
\hline Far UV & $10-100$ \\
\hline
\end{tabular}

It is known that the wavebands including the UVC and the Far UV produce little background radiation as these wavelengths are strongly absorbed by atmospheric elements [7]. The UVC region is often referred to as 'solar blind', as there is no background radiation in either day or night from the sun. This region is particularly useful for detecting very hot objects, such as missile plumes and flare $\mathrm{CM}$ as the energy from the sun cannot interfere with the detection and the only objects emitting in this region are very hot such as rocket plumes, CM flares. For this reason, it could be used to discriminate a hot $\mathrm{CM}$ object from the rest of a scene. However, this is negative discrimination, i.e. removing something undesirable. What the missile designer really wants is positive discrimination, i.e. keeping something desirable, the aircraft. For this reason, it would not be expected for a missile seeker to use UVC and Far UV. Limiting the missile seeker to the UVA and UVB is useful in the short wavelengths, which raises the question of whether a missile seeker could be configured to make use of any visible wavelength energy.

A representative ${ }^{i}$ UV scene of an aircraft with the sky as background is shown Figure 3. In this instance, the background level is low, uniform and positive with the dark aircraft object in the foreground creating a negative contrast. Owing to the low signature level of the background, the level of contrast in the picture is low. However, if the sky were white and the aircraft black, then this would be $100 \%$ contrast. This is what the missile seeker perceives; this scene allows discrimination of the aircraft from the background even in lower contrast situations. In this scene, a flare CM would appear as a bright spot that would also be easily discriminated from the background as well as the aircraft.

\footnotetext{
${ }^{\text {i }}$ This is not actually a UV scene, it is visible band scene, but it is illustrative of the UV and is the author's own photograph
} 


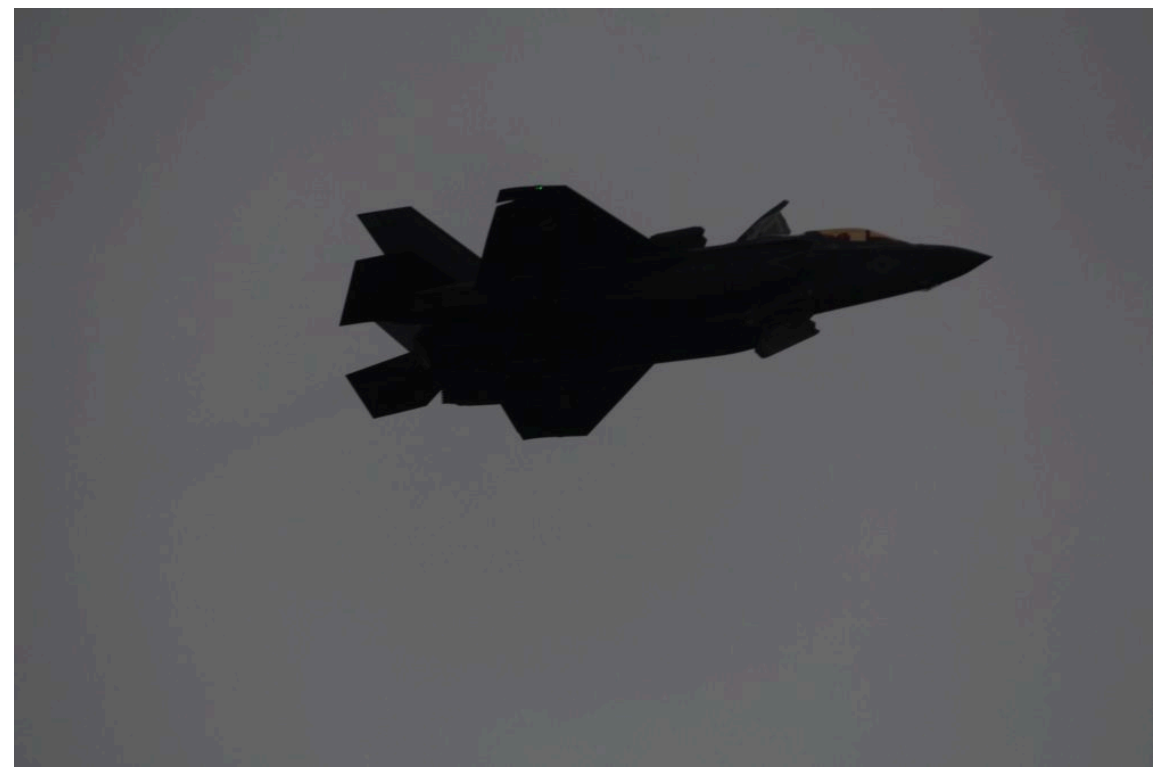

Figure 3 UV representative sky and aircraft scene

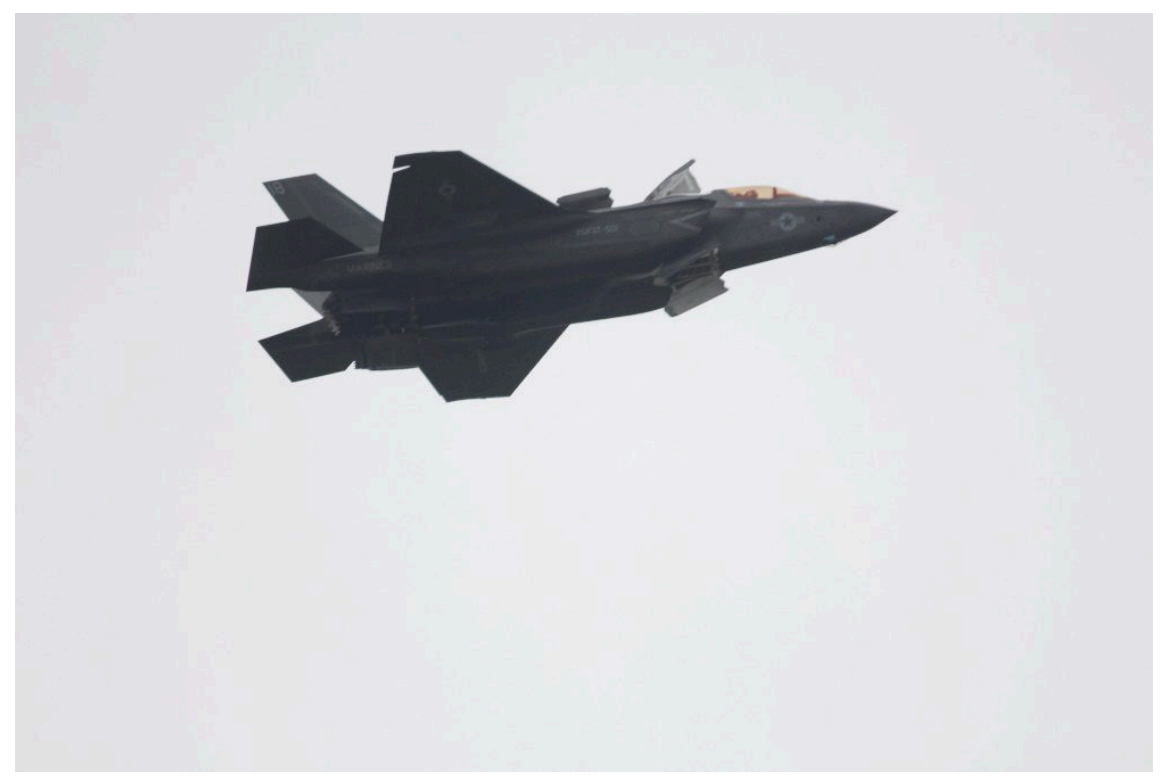

Figure 4 Visible sky and aircraft scene

Figure 4-shows the same scene taken one frame later but in the visible wavebands. Both positive and negative contrast can be seen between the aircraft and the sky. On the canopy particularly, the aircraft is bright against the grey sky background but, in other places, it is dark. This highly variable contrast is dependent on many factors, including the background colour, cloud cover, lighting direction, etc. Importantly for this study, empirical observations show that it is a feature that is much more unlikely to occur in the UV than in the visible. For this reason, a missile designer could choose to limit the detection waveband to include only the UVA and UVB, excluding any portion of the visible spectrum.

\subsection{The detector}

This appears to be a sensible assumption and further implies that the detector material could be Gallium based (Gallium Phosphate (GaP) or Gallium Arsenide Phosphide (GaAsP)) or Silicon ( $\mathrm{Si}$ ) as the response in these wavebands suitable as they all cover the $300-500 \mathrm{~nm}$ range. Si and GaAsP have a response that extends far into the visible wavebands; this 
would need to be filtered out to leave the UVA and UVB whereas GaP does not extend far into the visible wavebands [8].

\subsection{Other optical parameters}

Through application of analysis, some of the unknown aspects of the missile can be assumed. This is clearly important for modelling. Some other important assumptions include:

- The rosette petal pattern for the FN-16 is as per the arrangement assumed

- The SA-29 (Verba) slit arrangement is as per the data in the patent [1]

- The wavebands of the UV detector are limited to UVA and UVB

- The seekers will operate with a folded cassegrain telescope

- The seekers will have detectors which are comparable with commercially available ones

Some investigation was conducted into the likely Signal to Noise Ratio (SNR) within the missile optic. This SNR was defined as the loss that occurs within the missile seeker associated with the reduction in energy present at the missile optical dome compared to the energy present at the detector. This implicitly includes any loss associated with the optics in the missile seeker as well as the inherent noise within the detector material. There is no evidence of indicative figures for this SNR in the literature, as it is more common to be concerned with the SNR of the detector in isolation. However, often the detector in isolation can have an SNR in single figures that means that very little noise is inherent in the typical detectors used. Therefore, the majority of the SNR defined for this model must come from losses associated with the optical elements. Given this, in constructing the model to perform as the missile seeker is expected to, it was decided to vary this figure parametrically to determine the sensitivity of the system to variations as well as to identify a representative SNR for the rest of the testing. The selected initial SNR would give a maximum engagement range commensurate with typical shoulder launched EO SAM, particularly similar missiles. Internet references show that for the Stinger missile this is approximately $7-10 \mathrm{~km}[9]$.

\section{METHOD}

The results presented here are the first in a series of experiments and data gathering that will be conducted. The purpose of this section is to describe the parameters and the construction of the models that will be used within these experiments. The objective is to demonstrate that all of the relevant aspects of the missile can be represented.

The models of the missiles were built using Simulink ${ }^{\circledR}$ within Matlab ${ }^{\circledR}$. This allows for the monitoring of signals throughout the missile seeker as well as the commonality of various other ancillary components, such as the gyro, gimbal, rocket motor, warhead and guidance loop. These models of subcomponents were all taken from existing missile models that currently exist within the air platform protection (APP) community. A basic environment model was needed to model the attenuation of the signal reaching the missile that was variable with range. The data for the environmental model was taken from MODTRAN, which is a standard atmospheric transmission code [10]. Smith [11] showed that for specific meteorological conditions MODTRAN provides good estimation of the amount of UV radiation incident at the earth's surface. In terms of the model, the UV background radiation will be varied to represent various times of day and year, as well as different locations on the earth, various meteorological conditions and differing angles of elevation. Whilst MODTRAN is capable of calculating the background levels, other effects can alter the signature. One of these is the in-path scattering of UV radiation, which will be added through empirical methods, such as those calculated by Craig [12]. This approach will be supplemented with further empirical data before it is used in the models being created for this study.

The modelled missiles were presented with a target aircraft to track using their IR detector. The aircraft was given characteristics that ensured that it could be tracked at all times and at a wide variety of ranges. Its signature is not one that is associated with a particular aircraft but it is representative of a typical bright aircraft. The missile flies out towards the aircraft throughout the engagement, as there is no CM present in the initial testing. The only limitation on the engagement is kinematic, i.e. where the missile runs out of energy or maneuverability and so is unable to intercept the aircraft. These parameters are determined in the model by components taken from other missile models.

The UV detector will also monitor the presented scene so that the data from the UV detector can be interrogated to understand how it responds to the scene containing the aircraft and to determine whether the aircraft can be discriminated 
from the background. The missile will be launched from a range that ensures that the aircraft cannot be detected by the UV detector ensuring that the precise range at which the aircraft can be detected must be determined in post run processing. Some limited CCM functionality has been built into the missile model, although this will not be used in initial testing, given that there is no CM present. The aircraft is represented as an area of the scene that has low UV signal (set to zero initially). The signal level associated with the aircraft is varied to represent aircraft with different paint reflectivity. This is varied within the model by altering the contrast level between the aircraft and the sky background from the maximum (based on the initial conditions) to zero contrast. The data from the UV detector (or detectors) is recorded for each engagement and then is stored for post processing after the run is complete. Other data, such as the range from the missile to the aircraft, is recorded after the run for further processing and analysis. The UV intensity is plotted against range to determine whether the missile is functioning as expected.

Further analysis includes the addition of a noise level that must be exceeded to enable discrimination of the target object from the background. The detectivity of the detector has to be parametrically varied in addition to the SNR in order to ensure that the sensitivity to both is understood. The values of detectivity are estimated and are comparable to commercially available products [13].

The measure of performance to be reported is the range at which the UV detector is assessed to be able to discriminate the target aircraft from the background. This is the signal level at which the negative contrast pulse associated with the aircraft exceeds the background multiplied by the SNR level.

The values which may be changed within the model and for which the sensitivity of the results will be investigated are:

- The detectivity of the detector

- The SNR within the missile optics

- The cross sectional area of the aircraft target (a single dimension will be altered and assumed to be square)

- The reflectivity of the aircraft in the UV

- The speed and orientation of the target aircraft

\section{INITIAL RESULTS AND DISCUSSION}

The initial results indicated that there is correlation between the aircraft size and the range at which it can be detected. As the aircraft got larger in cross-section, so the range at which it could be detected increased. This is an expected result, as the solid angle subtended at the detector, by the aircraft at a particular range, will increase as the size of the aircraft increases. This increase in the size of the object will enable the contrast caused by it blocking the background energy to be discriminated at longer ranges. Working against this effect is the scattering of the UV energy. As the path length increases from the missile to the target, more of the UV energy from around the edge of the object is scattered into the viewing path. This blurs the edges of the object and decreases the contrast between the object and the background. The strength of the correlation between aircraft size and detection range is not linear. It is possible that this is owing to the cross-sectional area of the aircraft being increased, rather than to a linear variable.

A correlation was observed between the UV reflectivity of the aircraft surface and the range at which the aircraft could be detected. As the UV reflectivity increased, so the range at which the aircraft could be detected decreased. Again, this is an expected result. In theory, there is a maximum range at which a particular aircraft can be detected when the reflectivity is $0 \%$, which will be the limit of the detectivity of the detector at the highest contrast possible. The range of possible detection will tend to zero as the reflectivity of the aircraft tends to $100 \%$ as at this value, the contrast will be zero and the aircraft will not be able to be distinguished from the background at any range from the observer. Between these extremes, the relationship between the reflectivity of the aircraft and the range at which it can be detected will be defined.

At this stage of the research, these results show that the choices made for the variables and the modelling conducted are representative of the environment in which the threat is operating. Closer examination of the signals produced by the missile seeker indicates that the IR and UV channel are producing the sorts of signals that would be expected. Therefore, the missile is able to track the aircraft in the IR (for the missile that has been successfully modelled and tested) Overall, it is assessed that the models and the environment are broadly representative and can be built upon to investigate what further. Initial results indicate that the assumptions made are valid and that the models that have been created are broadly 
valid. Therefore, the next stage in this study is to finish the model of the second missile and conduct further testing of the sensitivities of the results to the variables within the model.

\section{CONCLUSIONS}

This experiment has determined the likely wavebands that a missile seeker operating in the UV will use as well as building basic models of two specific EO SAM missile seekers that use a UV detector. It has proven the concept that models of the missile seekers as well as the UV scene can be produced and used. Understanding of the UV scene in which these detectors operate has been developed and modelled. The missiles models that have been produced exhibit some unique features, which have made the models more complex and have required deeper understanding of the particular aspects of the designs. It appears from this experiment that both the environment and the missiles can be modelled well enough to produce typical results as expected. This confirms the viability of this approach to the problem of determining the likely characteristics and the performance of potential threat missiles.

Further work will include the transfer of the model from one network to another, continuity checking and generation of further results for the single missile for which testing has been conducted to generate further results in more atmospheric, treatment and range conditions. The second missile model will also be finished and all of the tests will be repeated using the new model to investigate the sensitivity of the results to the missile architecture chosen. Clearly, a comparison of one missile type to the other should also be completed where differences exist in the results that they produce.

The required level of reflectivity that an aircraft needs to limit the effectiveness of the missile seeker will be determined for each missile modeled. This will vary depending on the acceptable range at which the UV detector can be allowed to discriminate the aircraft from the background and on the aircraft for which the data is required. Initial results indicate that there is value in controlling the aircraft signature to limit the effectiveness of a missile seeker operating with a detector in the UV.

Subsequent work will include the addition of CM testing against the modelled missile seekers to determine what sort of $\mathrm{CM}$ would be most effective. Validation of the modelling technique and the representation of the background should be conducted with practical representations wherever possible. Clearly, for the missile seekers this will be difficult as access to the missile seekers for comparison to the model is unlikely to be granted. However, it should be possible to check that the scene used is representative. In all cases, the sensitivity of the result to the chosen variables should be observed to ensure robust advice is given to others within the APP community who will seek to protect aircraft from these types of missiles.

\section{REFERENCES}

[1] A. A. Anishkin, V.P., Shibayev, A.K., Pomeranets, Ye.Ya., Semonovskiy, B.N., Prutt, A.M., Gurevich, M.S., Chekin, V.M. \& Tsymlov, "Optical-electronics seeker," 2000.

[2] M. S. Gurevich, V. N. Eskin, A. M. Chuprakov, and N. Y. Shustov, "Trispectral target seeker for an optical homing head," J. Opt. Technol., vol. 69, no. 9, pp. 648-651, 2002.

[3] T. Tajime, S. Wakabavashi, M. Kondo, T. Takei, E. Mitsubishi, and R. N. Hubbard, "Rosette scan infrared sensor," in 1980 Los Angeles Technical Symposium, 1980, pp. 51-57.

[4] S. Jahng, H. Hong, S.-H. Han, J. S. Choi, S. Jahnga, H. Honga, S.-H. Han, and J. S. Choi, "Design of Instantaneous Field of View of the Rosette Scanning Infrared Seeker and Dynamic Simulation," SPIE Conf. Autom. Target Recognit., vol. 3365, no. April 1998, pp. 158-168, Jul. 1998.

[5] T. W. Tucker, "Evaluating Airliner MANPADS Protection,” no. 613, 2009.

[6] R. P. Birchenall, "Modelling Infrared Man Portable Air Defence Systems to Develop Infrared Countermeasures," Cranfield University, 2011.

[7] E. Trakhovsky, A. Ben-Shalom, U. P. Oppenheim, A. D. Devir, L. S. Balfour, and M. Engel, "Contribution of oxygen to attenuation in the solar blind UV spectral region.," Appl. Opt., vol. 28, no. 8, pp. 1588-91, Apr. 1989.

[8] V. Gonzlez, D. Barrientos, J. M., F. Carri, X. Egea, and E. Sanchis, "Data Acquisition in Particle Physics 
Experiments," in Data Acquisition Applications, InTech, 2012.

[9] M. S. M. Systems and L. A. Defence, "European Stinger-RMP,” vol. 1, no. September 1997, pp. 1-3, 2013.

[10] D. Zondervan and M. A. Supervisor: Richardson, "IR countermeasures for a large jet aircraft in a simulated MANPAD engagement," Cranfield University, 2012.

[11] L. Smith, M. Richardson, and R. Walmsley, "Comparison of MODTRAN5 to measured data in the UV band," in SPIE Security + Defence, 2013, vol. 8898, p. 88980F.

[12] B. Craig, "Atmospheric attenuation of Ultraviolet Contrast," 2016.

[13] "Unmounted Photodiodes." [Online]. Available:

https://www.thorlabs.de/newgrouppage9.cfm?objectgroup_id=285. 